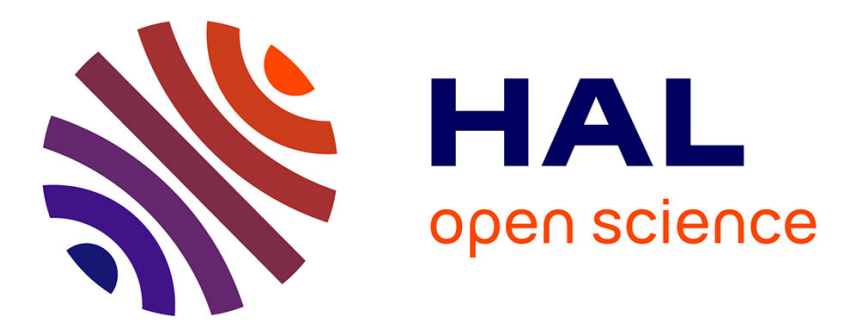

\title{
Propriétés magnétiques de films métalliques ultra-minces : détermination de la structure électronique
}

O. Elmouhssine, M. Freyss, D. Stoeffler, A. Vega, G. Moraitis, C. Demangeat, H. Dreyssé

\section{- To cite this version:}

O. Elmouhssine, M. Freyss, D. Stoeffler, A. Vega, G. Moraitis, et al.. Propriétés magnétiques de films métalliques ultra-minces: détermination de la structure électronique. Journal de Physique IV Proceedings, 1996, 06 (C7), pp.C7-225-C7-232. 10.1051/jp4:1996729 . jpa-00254517

\section{HAL Id: jpa-00254517 https://hal.science/jpa-00254517}

Submitted on 1 Jan 1996

HAL is a multi-disciplinary open access archive for the deposit and dissemination of scientific research documents, whether they are published or not. The documents may come from teaching and research institutions in France or abroad, or from public or private research centers.
L'archive ouverte pluridisciplinaire HAL, est destinée au dépôt et à la diffusion de documents scientifiques de niveau recherche, publiés ou non, émanant des établissements d'enseignement et de recherche français ou étrangers, des laboratoires publics ou privés. 


\title{
Propriétés magnétiques de films métalliques ultra-minces: détermination de la structure électronique
}

\author{
O. Elmouhssine, M. Freyss, D. Stoeffler, A. Vega*, G. Moraitis, C. Demangeat et H. Dreyssé \\ Institut de Physique et Chimie des Matériaux de Strasbourg, Université Louis Pasteur, 23 Rue du Loess, \\ 67037 Strasbourg, France \\ * Departamento de Física Teórica, Universidad de Valladolid, Valladolid, Spain
}

\begin{abstract}
Résumé: L'étude des systèmes artificiels à une échelle atomique est devenue un domaine de recherche très actif. Les propriétés magnétiques sont particulièrement étudiées pour leurs applications technologiques. Ces travaux posent aussi des questions fondamentales. Dans cette contribution deux exemples permettront d'illustrer la richesse des recherches actuellement menées et de présenter certaines approches théoriques et numériques utilisées pour décrire ces systèmes et notamment leur structure électronique à $\mathrm{T}=0 \mathrm{~K}$. Le cas d'une monocouche de $\mathrm{Mn}$ sur $\mathrm{Fe}(001)$ pose le problème de la possibilité de plusieurs arrangements magnétiques. Par un calcul TB-LMTO, en envisageant 5 différents types d'ordre 2D, il est trouvé que deux arrangements quasi-dégénérés permettent de décrire les résultats expérimentaux. La croissance d'un film polarisé sur un substrat, lui aussi polarisé, pose d'importantes questions. Dans le cas traité ici de la croissance du Fer sur le Chrome (001), un calcul de la structure électronique permet de comprendre les résultats expérimentaux s'il est tenu compte d'interfaces non parfaites présentant des défauts.
\end{abstract}

\section{INTRODUCTION}

L'étude, tant expérimentale que théorique, de matériaux artificiels mobilise un nombre toujours croissant de chercheurs de part le monde. Les propriétés magnétiques sont particulièrement étudiées. La vitalité de ce domaine de recherche est attestée par le nombre de conférences et "workshops" organisés sur ces thématiques [1] et les nombreuses collaborations entre groupes, soutenues notamment par des projets européens. Sans nul doute les applications technologiques possibles de ces matériaux stimulent cet axe de recherche. La mise en évidence d'une Magnéto Résistance Géante dans les multicouches $\mathrm{Fe} / \mathrm{Cr}$ par l'équipe de A. Fert à Orsay en 1988 [2] a permis la réalisation de capteurs très sensibles. La possibilité que peuvent avoir certains films très minces (d'une épaisseur de quelques plans atomiques) de présenter une aimantation perpendiculaire à la surface ouvre la voie à un accroissement considérable des performances d'enregistrement magnétique. Une ère nouvelle que certains ont qualifiée de "magnétoélectronique" s'ouvre [3].

Ces résultats interpellent les théoriciens. Les difficultés rencontrées pour décrire correctement le comportement magnétique de matériaux sous une forme massive (prédiction des températures de Curie, par exemple) se trouvent multipliées quand les propriétés magnétiques sont gouvernées par un arrangement à l'échelle atomique. Ainsi dans les tricouches $\mathrm{Fe} / \mathrm{Cr} / \mathrm{Fe}$, une interface plus ou moins 
parfaite associée à des températures de préparation des échantillons différentes conduit à une courte ( 2 monocouches) ou à une longue (10-12 monocouches) période d'oscillation du couplage d'échange [4]. De nombreux résultats expérimentaux contradictoires ont été reportés. Nous en donnerons deux exemples dans cette contribution et essayerons de montrer que les travaux théoriques peuvent permettre "d'y voir plus clair".

Dans cette contribution nous nous focaliserons sur les propriétés magnétiques de systèmes à base de métaux de transition à $\mathrm{T}=0 \mathrm{~K}$. D'une façon générale il est bien connu que les propriétés magnétiques sont dues aux électrons. La détermination de la structure électronique par un formalisme adéquat conduit à la distribution des moments magnétiques locaux. De plus si le couplage spin-orbite est introduit, l'orientation de l'aimantation dans l'espace est connue. Dans les métaux, les électrons ne sont plus "attachés" à des atomes précis et le magnétisme itinérant est le cadre idéal pour décrire ces systèmes. Le développement de la théorie de la fonctionnelle de densité [5] et son extension aux systèmes polarisés et relativistes a été décisif. Néanmoins ces approches se heurtent à la difficulté récurente qui est que la "vraie" fonctionnelle en fonction de l'énergie d'échange et de corrélation n'est pas connue. A ce niveau des approximations sont nécessaires. La plus courante est dite de la Densité Locale (LDA) et donne de bons résultats. Ses limitations sont connues. Le Fer est certainement l'exemple le plus probant: le meilleur calcul LDA prédit un état de base cubique faces centrées [6]; il faut améliorer les termes d'échange-corrélation par des corrections de gradient pour trouver comme état d'énergie minimale la structure cubique centrée [7].

D'une façon schématique, deux types d'approches sont utilisées pour déterminer la structure électronique [8]. Les méthodes dites ab-initio conduisent aux résultats les plus précis; le prix à payer en sont des calculs très lourds qui ne s'appliquent qu'à un petit nombre de sites atomiques inéquivalents et qui en général demandent une certaine symétrie de translation $[9,10]$. D'un autre coté les méthodes semi-empiriques comme les liaisons fortes permettent d'aborder l'étude de systèmes complexes inaccessibles sinon $[8,11]$. Un effort important de recherche porte actuellement sur des versions "liaisons fortes" (TB) des méthodes ab-initio, que ce soit pour des approches TB-LMTO $[12,13]$ ou TB-KKR [14]. On obtient ainsi des méthodes d'ordre $\mathrm{N}$ qui allient la précision des méthodes ab-initio et les possibilités offertes par des méthodes de liaisons fortes (études de systèmes complexes et/ou systématiques ...).

\section{FILMS ULTRA-MINCES}

Il est maintenant bien établi que les films ultra-minces peuvent présenter des propriétés magnétiques très différentes de celles qu'ils pourraient avoir dans un état massif. Du fait de la portée limitée des interactions interatomiques, en général, au delà d'une épaisseur de 1 à 2 nanomètres le film a un comportement massif; seuls les plans près des interfaces ou des surfaces libres ont des moments magnétiques différents de ceux au centre du film. Néanmoins dans le cas d'interfaces non parfaitement planes et/ou en présence de défauts induisant des frustrations magnétiques [15], la zone perturbée peut être plus importante.

Le cas le plus simple, en apparence, est celui d'une monocouche parfaite déposée sur un substrat. D'un point de vue théorique, cette situation a été étudie par diverses méthodes $[16,17,18,8]$. En général trois configurations magnétiques sont envisagées: un ordre ferromagnétique dans le plan avec deux types de couplage avec le substrat (les configurations $\mathrm{p}(1 \times 1) \uparrow$ et $\mathrm{p}(1 \mathrm{x} 1) \downarrow$ de la figure $1 \mathrm{a}$ ) et un ordre antiferromagnétique dans le plan (configuration $c(2 \times 2)$ de la figure 1a). Dans le cas d'un substrat non polarisé comme un métal noble les deux configurations $\mathrm{p}(1 \times 1)$ sont identiques. Plusieurs travaux $[19,20]$ ont montré que les métaux de transition en bout de série (comme le Ni) "préfèrent" un couplage Parallèle entre premiers voisins et ceux du milieu de série un couplage Antiparallèle. De plus la symétrie particulière de la monocouche rend la solution $c(2 \times 2)$ très favorable pour les éléments de milieu de série. Ceci a été confirmé expérimentalement dans le cas d'une monocouche de Cr sur Ag(001) [21] par exemple. 
a)

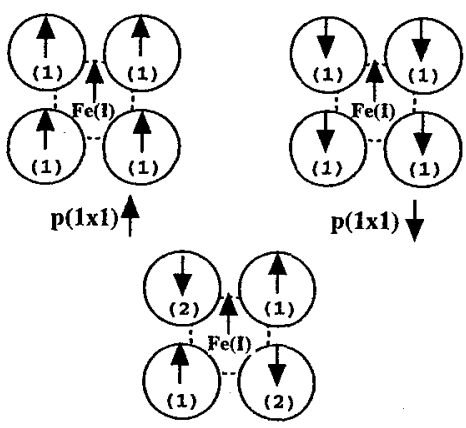

$\mathbf{c}(2 \times 2)$

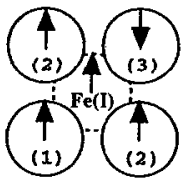

$\mathrm{p}(2 \times 2) \uparrow$

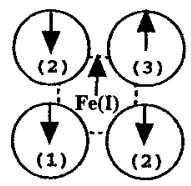

$p(2 \times 2) \frac{1}{\gamma}$ b)

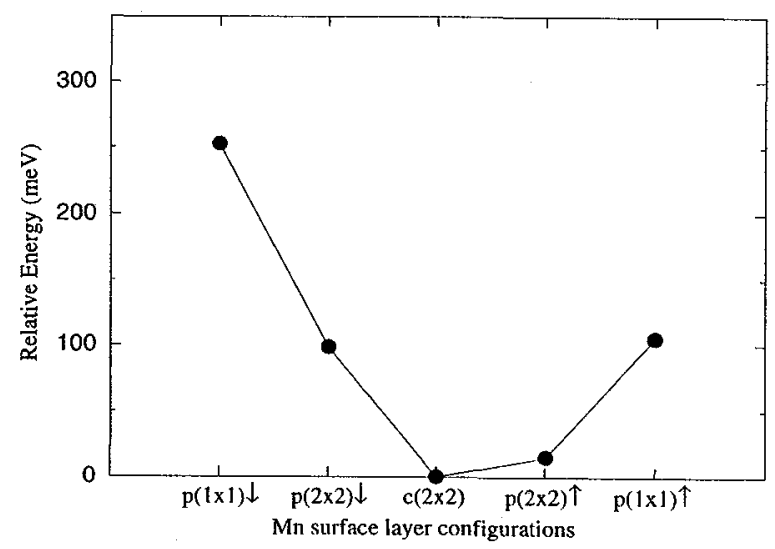

Figure 1: a) Vue schématique de 5 configurations magnétiques possibles d'une monocouche de $\mathrm{Mn}$ sur du Fe(001). Les cercles pleins (pointilllés) correspondent aux atomes de $\mathrm{Mn}$ ( $\mathrm{Fe}$ ). Les flèches indiquent la direction des moments locaux [29]. b) Energie de chaque configuration, définie en 1a; la référence étant prise pour l'arrangement c(2x2) [29].

Le cas d'un film mince de Mn est particulièrement intéressant. En effet le Manganèse est connu pour des arrangements magnétiques complexes [22]. On peut donc s'attendre à ce qu'il en soit de même dans le cas d'une monocouche. Par spectroscopie de photoélectrons de coeur polarisés, Roth et al. [23] ont observé une configuration $c(2 \times 2)$ de la couche de $\mathrm{Mn}$, avec un moment magnétique moyen proche de zéro $\left(0.3 \mu_{B}\right)$ et antiparallèle au moment de Fe, en accord avec divers travaux théoriques [24, 25, 26]. Mais une étude très récente [27] en dichrö̈sme magnétique a conduit à l'existence d'un ordre qui ne pouvait être $c(2 \times 2)$ et que ces auteurs ont supposé $p(2 \times 2) \uparrow$. Pour des films d'épaisseur jusqu'à 2 monocouches (ML) un moment magnétique global, estimé à $1.7 \mu_{B}$ par atome de $\mathrm{Mn}$, a été observé. Les différences observées entre ces deux travaux semblent être dues à des modes de préparation différents. Andrieu et al. [27] ont fait croitre le film de Mn sur un buffer de 200 plans de $\mathrm{Fer}$, déposé lui-même sur un substrat de $\mathrm{MgO}$, alors que Roth et al. [23] ont considéré un film de Fer beaucoup plus mince (20 plans) déposé sur un substrat d'Ag(001). Mais pour ces sytèmes il y a alors ségrégation de l'Argent, ce qui conduit à un tout autre arrangement atomique et donc à une distribution différente des moments magnétiques locaux [28]. On ne saurait parler de résultats expérimentaux antagonistes puisqu'en fait il s'agirait alors de deux systèmes présentant un arrangement chimique différent et donc des propriétés magnétiques différentes.

Pour comprendre cette situation expérimentale, Elmouhssine et al. [29] ont calculé à l'aide de la méthode TB-LMTO la distribution des moments magnétiques d'une monocouche parfaite de Mn sur du $\mathrm{Fe}(001)$. Aux trois types d'arrangement magnétiques déjà mentionnés, ont été ajoutés deux autres arrangements notés $\mathrm{p}(2 \times 2)$, comme indiqué dans la figure 1a. Dans tous les cas, les quatre atomes de Mn de la maille portent un moment magnétique de l'ordre de $3 \mu_{B}$. Pour la configuration $\mathrm{p}(2 \times 2) \uparrow$ trois de ces atomes de $\mathrm{Mn}$ ont un moment parallèle à ceux du Fer et le quatrième un moment antiparallèle, alors que pour la structure $\mathrm{p}(2 \times 2) \downarrow$ la situation est inversée. Comme l'indique la figure 
$1 b$, ces calculs montrent que les deux configurations $\mathrm{c}(2 \times 2)$ et $\mathrm{p}(2 \times 2) \uparrow$ sont quasi dégénérés. L'étude détaillée des moments magnétiques obtenus pour ces cinq configurations (tableau 1) suggère que la valeur du moment magnétique global observé par Andrieu et al. [27] correspond à l'arrangement $\mathrm{p}(2 \times 2) \uparrow$; le moment moyen trouvé de $1.59 \mu_{B}$ est proche de la valeur expérimentale. Ce calcul $a b$ initio a été effectué dans l'approximation dite ASA (Atomic Sphere Approximation) qui ne permet pas une relaxation des atomes de la monocouche; seule une étude utilisant un cadre FP (Full Potential) permettrait d'étudier une éventuelle "ondulation" de la monocouche et l'effet des relaxations perpendiculaires à la surface sur la valeur des moments magnétiques. Néanmoins les ordres de grandeur des différences d'énergie permettent d'affirmer que les deux états devraient rester d'énergie très proche.

Table 1: Distribution des moments magnétiques locaux d'une monocouche de Mn sur Fe(001) pour 5 configurations (Cf fig. 1a). Les nombres entre parenthèses pour $\mathrm{Fe}(\mathrm{I}-1)$ dans l'état $\mathrm{c}(2 \times 2)$ correspondent au moment de l'atome sous $\mathrm{Mn}(2)$ et dans les états $\mathrm{p}(2 \times 2)$ aux moments des atomes sous $\mathrm{Mn}(2)$ et $\mathrm{Mn}(3) . \mathrm{M}_{S}$ est l'aimantation moyenne de surface (d'après [29]).

\begin{tabular}{|l||c|c|l|r|r|r|r||r|}
\hline $1 \mathrm{Mn} / \mathrm{Fe}(001)$ & $\mu_{\mathrm{Fe}(\mathrm{C})}$ & $\mu_{\mathrm{Fe}(\mathrm{I}-2)}$ & $\mu_{\mathrm{Fe}(\mathrm{I}-1)}$ & $\mu_{\mathrm{Fe}(\mathrm{I})}$ & $\mu_{\mathrm{Mn}(1)}$ & $\mu_{\mathrm{Mn}(2)}$ & $\mu_{\mathrm{Mn}(3)}$ & $M_{S}$ \\
\hline$p(1 \times 1) \downarrow$ & 2.20 & 2.23 & 2.38 & 1.46 & -3.15 & -3.15 & -3.15 & -3.15 \\
$p(1 \times 1) \uparrow$ & 2.24 & 2.26 & 2.24 & 1.61 & 3.42 & 3.42 & 3.42 & 3.42 \\
$p(2 \times 2) \downarrow$ & 2.20 & 2.25 & $2.33(2.33 / 2.33)$ & 1.41 & -3.01 & -3.12 & 2.95 & -1.58 \\
$p(2 \times 2) \uparrow$ & 2.20 & 2.25 & $2.38(2.26 / 2.10)$ & 1.40 & 3.30 & 3.23 & -3.37 & 1.59 \\
$c(2 \times 2)$ & 2.19 & 2.23 & $2.27(2.35)$ & 1.27 & 3.13 & -3.34 & 3.13 & -0.10 \\
\hline
\end{tabular}

Un certain nombre de remarques peuvent être faites sur les valeurs des moments magnétiques locaux. Comme déjà indiqué ceux-ci sont de l'ordre de $3 \mu_{B}$ indépendemment du type de couplage (parallèle ou antiparallèle) entre eux. C'est une tendance générale notée dans d'autres calculs [8]. On peut, néanmoins, remarquer que les valeurs les plus importantes sont obtenues pour la configuration $\mathrm{p}(1 \mathrm{x} 1) \uparrow$ qui correspond à un arrangement où tous les atomes ont un moment parallèle. Les valeurs absolues des moments diminuent au fur et à mesure que l'on "inverse" le sens des atomes de Mn (passant des configurations $\mathrm{p}(1 \times 1) \uparrow$ à $\mathrm{p}(2 \times 2) \uparrow$, puis $\mathrm{c}(2 \times 2)$ et $\mathrm{p}(2 \times 2) \downarrow)$. Par contre la configuration $p(1 \times 1) \downarrow$, qui correspond aux 4 atomes de Mn "inversés" ne suit pas cette tendance. Ceci est, probablement, due à l'absence de frustrations magnétiques: tous les couplages Mn-Fe sont antiparallèles. On retrouve un comportement qui a été observé dans d'autres systèmes comme Fe/Cr: les frustrations magnétiques ont tendance à faire décroître les moments magnétiques locaux [15]. Il faut quand même rappeller que le calcul complet de tous les moments magnétiques locaux est absolument nécessaire; ce n'est qu'à partir de ces résultats qu'une interprétation en termes de tendances générales peut être faite et non l'inverse.

\section{CROISSANCE DU CHROME SUR LE FER}

Depuis la mise en évidence d'un couplage Antiferromagnétique [30] et d'une Magnéto Résistance Géante [2] dans les multicouches $\mathrm{Fe} / \mathrm{Cr}$, le système $\mathrm{Fe} / \mathrm{Cr}$ est devenu probablement le système le plus étudié [31]. Dans la richesse des problèmes abordés, nous en retiendrons un en particulier. La croissance du Fer sur le Chrome a fait l'objet de plusieurs travaux expérimentaux aux conclusions différentes. En déposant du Fe sur du Cr lui-même déposés sur une surface de Fe(001) présentant de 
nombreuses facettes, l'aimantation globale ne varie pas sur les premières monocouches (fig. 2) pour augmenter de façon linéaire par la suite [32]. Il semble donc que la qualité de l'interface joue un rôle important et que l'hypothèse communément retenue par les théoriciens d'une interface parfaitement plane ne soit qu'une description approchée des systèmes "réels".

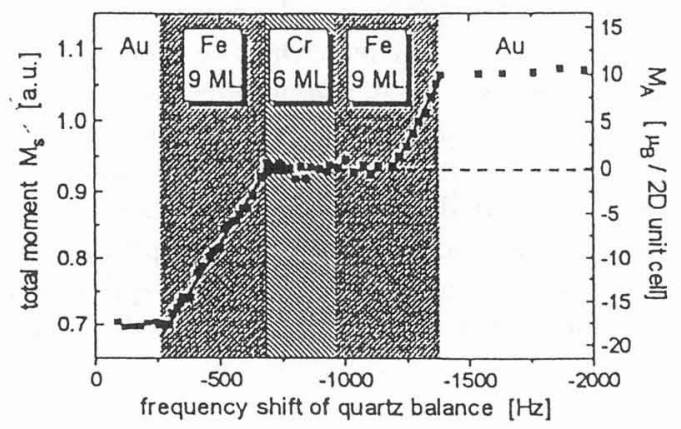

Figure 2: Aimantation spontanée d'un film obtenu par déposition successive de Fer, de Chrome et de Fer à nouveau sur un substrat d'Au(001) présentant de nombreuses facettes. En abscisse est donnée la variation de l'aimantation totale normalisée au nombre d'atomes de la surface. Résultat expérimental de Miethaner et Bayreuther [32].

Pour étudier l'influence d'une interface non abrupte sur la répartition des moments magnétiques, divers types de défauts ont été envisagés dans un calcul récent de liaisons fortes [15]. Un des résultats les plus marquants est que, du grand nombre de solutions numériques, deux solutions émergent dans le cas de "vallées" ou de "bosses". La figure 3 illustre ces deux configurations dans le cas d'une "vallée".

a)
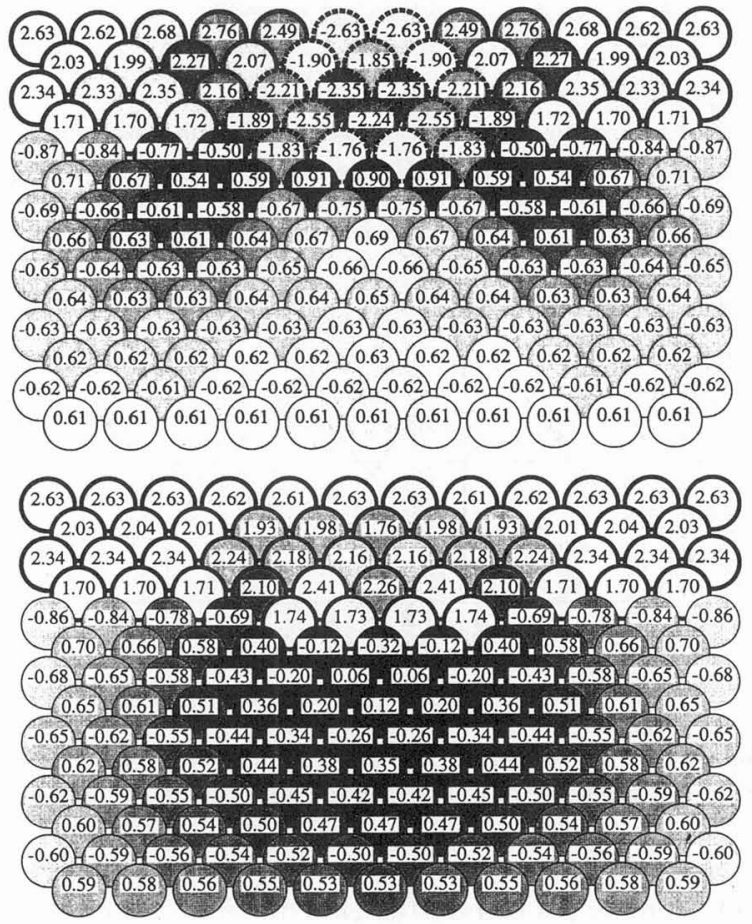

Figure 3: Distribution de moments magnétiques locaux près d'une vallée à l'interface $\mathrm{Fe} / \mathrm{Cr}$ (les atomes de Fer sont entourés d'un cercle noir) par projection sur un plan (010). Deux arrangements sont en compétition: le film de Fer présente a) plusieurs domaines magnétiques (MD); b) un seul domaine (SD) [15]. 
Une première solution correspond à un substrat de Chrome peu affecté par le film de Fer. La présence de ce dernier induit une augmentation du moment magnétique local des atomes de Cr près de l'interface mais tous les atomes dans des plans (001) ont un même signe de polarisation. La topologie de ces sytèmes imposent des frustrations magnétiques, au sens qu'il est impossible d'avoir une configurations de moments colinéaires où tous les couplages $\mathrm{Cr}-\mathrm{Fe}$ et $\mathrm{Cr}-\mathrm{Fe}$ ( $\mathrm{Fe}-\mathrm{Fe}$ respectivement) entre premiers voisins soient Antiparallèles (Parallèles). Dans ce premier cas la persistance de l'ordre antiferromagnétique dans le Chrome impose l'existence de plusieurs domaines magnétiques pour le film de Fer (tous les atomes dans un plan (001) n'ont pas la même direction de polarisation ( fig. 3a )). La deuxième solution correspond à un film de Fer monodomaine. La conséquence en est une très forte perturbation dans le substrat de Cr près de l'interface où les moments magnétiques voient leur valeur fortement diminuer. L'ampleur spatiale de cette perturbation est remarquable (fig. $3 b$ ). Une étude détaillée de ces résultats montre, à l'instar de ce qui avait été mis en évidence au paragraphe précédent pour $\mathrm{Mn} / \mathrm{Fe}$, que les frustrations magnétiques diminuent la valeur des moments magnétiques.

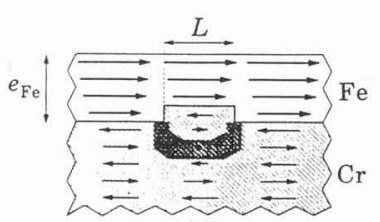

SD

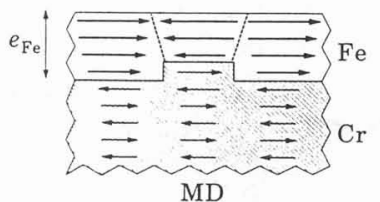

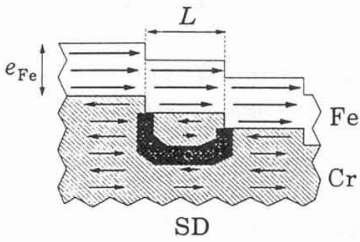

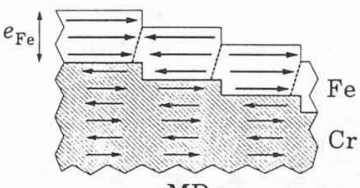

$\mathrm{MD}$

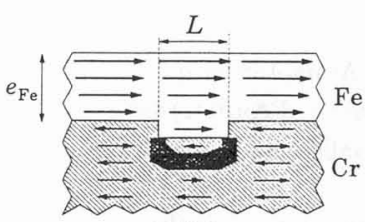

$\mathrm{SD}$

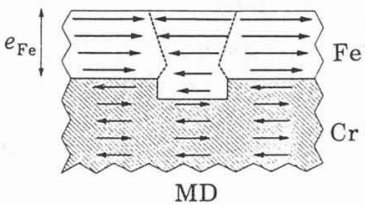

Figure 4: Vue schématique des arrangements magnétiques obtenus pour trois types d'irrégularité à l'interface $\mathrm{Fe} / \mathrm{Cr}$ et les deux types d'ordre magnétique du Fer (SD et MD comme défini sur la figure 3) [15].

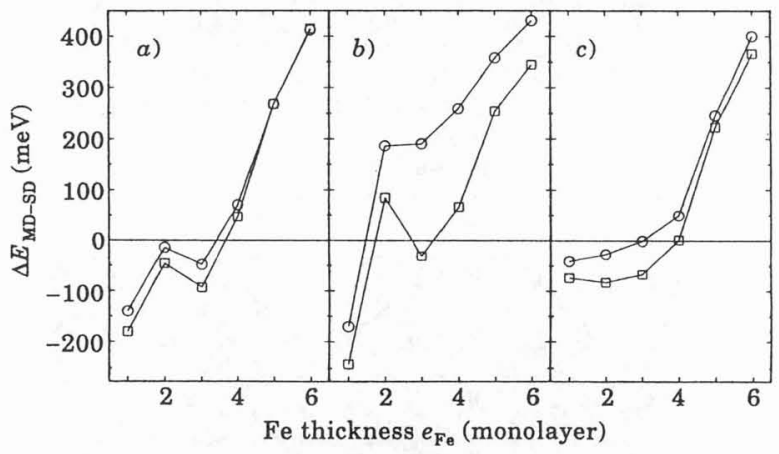

Figure 5: Différence d'énergie entre les solutions SD et MD en fonction de l'épaisseur du film de Fer pour différents types d'irrégularité à l'interface $\mathrm{Fe} / \mathrm{Cr}$ : a) bosse; b) marche et c) vallée. Les cercles (carrés) correspondent à des irrégularité de 3 (4) colonnes. Des valeurs positives (négatives) de l'énergie indiquent que la solution SD (MD) est la plus stable [15].

Sur la figure 4 sont reportés les comportements dus à des interfaces rugueuses. Dans les trois cas étudiés les deux solutions domaines simples (SD) ou multiples (MD) sont trouvées. Les deux grandeurs caractéristiques sont la largeur du défaut et l'épaisseur du film de Fer. Une étude pour une largeur de 3 et 4 rangées atomiques (fig. 5) met en évidence qu'au delà d'une certaine épaisseur de 
Fer, le film de Fer présente un domaine unique. Intuitivement ceci se comprend aisément par le fait que l'on a un nombre fini de frustrations à l'interface et que par contre le nombre de frustrations dans le film de Fer à la limite des domaines augmente de façon linéaire avec l'épaisseur de Fer. Néanmoins le calcul complet des différences d'énergie totale montre que celles-ci ne varient pas de façon linéaire.

Ces résultats permettent d'interpréter les résultats expérimentaux reportés sur la figure 2. Au début de la croisance du Fer sur le Cr, il y a de nombreuses irrégularités à l'interface, le Fer présente des domaines et, en moyenne, l'aimantation est nulle. Par contre au delà de $5 \mathrm{ML}$ il acquiert progressivement une structure en domaine unique. D'une façon générale, ces travaux mettent en évidence qu'au delà d'une épaisseur de Fer qui est de l'ordre de grandeur de la taille latérale des défauts, il y a homogénisation de l'aimantation du Fer, les défauts sont en quelque sorte "guéris" [33]. Dans le cas de biseaux de Fer sur Cr, la taille importante des marches successives est bien plus grande que l'épaisseur de Fer ce qui permet d'observer des domaines clairement définis et par là même la nature du couplage des couches de Fer au travers du film de Chrome [4].

\section{CONCLUSIONS ET PERSPECTIVES}

Dans cet article ont été présentés deux situations expérimentales différentes: les ordres magnétiques possibles d'une monocouche (Mn sur Fe(001)) et la croissance du Fer sur le Chrome. Dans ces deux cas a été mis en évidence la nécessité de réaliser un calcul auto-cohérent complet de la structure électronique. D'une façon phénoménologique seules des tendances générales peuvent être proposées. Un des enjeux dans ce domaine de recherche est de pouvoir augmenter encore la taille des problèmes étudiés pour reproduire les situations expérimentales de façon satisfaisante. Les études par Dynamique Moléculaire "à partir des premiers principes" devraient apporter des réponses intéressantes.

Dans cet article, seules des orientations de moments colinéaires ont été envisagées. Divers résultats expérimentaux suggèrent que les ordres non-colinéaires peuvent être engendrés par des défauts structuraux et sont donc largement présents. Un effort important de recherche porte sur l'étude d'arrangements non-colinéaires [34,35]. De même l'étude de l'anisotropie magnétique, c'est à dire de la direction dans l'espace physique de l'aimantation, n'a pas été considérée. Pour cela il faut introduire le couplage spin-orbite. Ce sont des calculs très délicats car les différences d'énergie entre deux directions de l'aimantation sont très faibles (inférieures au meV). L'effort important de recherche dans ce domaine devrait encore s'amplifier dans un futur proche [36].

\section{Remerciements}

Ce travail a été partiellement réalisé dans le cadre du Réseau Européen HCM $\psi_{\mathbf{k}}$, du Réseau Européen TMR "Interface Magnetism" et du programme de collaboration franco-allemand PROCOPE. Nous voudrions remercier en particulier G. Bayreuther et S. Miethaner de Regensburg pour de précieuses discussions des résultats expérimentaux sur l'interface $\mathrm{Fe} / \mathrm{Cr}$. L'Institut de Physique et de Chimie des Matériaux de Strasbourg est une Unité Mixte Associée au CNRS n 46.

\section{References}

[1] Voir par exemple les proceedings de la conférence annuelle "Magnetism et Magnetic Materials" ( J. of Appl. Phys. 154 (1996) ou ceux du "Second International Symposium on Metallic Multilayers" (J. Magn. Magn. Mat. 156 (1996)) 
[2] M.N. Baibich et al., Phys. Rev. Lett. 61 (1988) 2472; A. Barthelemy, A. Fert, R. Morel et L. Steren, Physics World (November 1994) 34

[3] Voir par exemple dans la livraison d'avril 1995 de Physics Today, pages 24 et suivantes

[4] J. Unguris, R.J. Celotta, et D.T. Pierce, Phys. Rev. Lett. 67, 140 (1991)

[5] P. Hohenberg et W. Kohn, Phys. Rev. 136 (1964) B864; W. Kohn et L. J. Sham, Phys. Rev. 140 (1965) A1133

[6] D. Singh, D. Clougherty, J. MacLaren, R. C. Albers and C. Wang, Phys. Rev. B 44 (1991) 7701

[7] D. Singh, W. Pickett et H. Krakauer, Phys. Rev. B 43 (1991) 11628

[8] H. Dreyssé et C. Demangeat, Surf. Sci. Rep. (1997)

[9] M. Weinert et S. Blügel in Magnetic Multilayers (eds. L. H. Bennett et R. E. Watson), World Scientific, Singapore (1994), 51

[10] S. Blügel et C. Carbone in Fundamental aspects of thin film magnetism (eds., A. Rettori et D. Pescia), World Scientific, Singapore (1996)

[11] Voir par exemple la contribution de M.C. Desjonquères et D. Spanjaard dans cet ouvrage

[12] O. K. Andersen et B. Jepsen, Phys. Rev. Lett. 53 (1984) 2571

[13] R. Lorenz et J. Hafner, J. Phys.:Condens. Matter 18 (1995) L253

[14] R. Zeller, P. H. Dederichs, B. Ujfalussy, L. Szunyogh et P. Weinberger, Phys. Rev. B 52 (1995) 8807

[15] A. Vega, D. Stoeffler, H. Dreyssé and C. Demangeat, Europhys. Lett. 31 (1995) 561

[16] S. Blügel, M. Weinert et P. H. Dederichs, Phys. Rev. Lett. 60 (1988) 1077

[17] S. Blügel, Solid St. Comm. 84 (1992) 621; Europhys. Lett. 18 (1992) 257; Phys. Rev. Lett. 68 (1992) 851

[18] S. Mirbt, O. Eriksson, B. Johansson et H. L. Skriver, Phys. Rev. 52 (1995) 15070

[19] V. Heine et J. H. Samson, J. Phys. F 13 (1983) 2155

[20] D. Stoeffler et H. Dreyssé, Solid State Comm., 79 (1991) 645; D. Stoeffler,G. Fabricius, A.M. Llois et H. Dreyssé, Surf. Sci. 269/270 (1992) 632; D. Stoeffler, non publié.

[21] C. Krembel, M.C. Hanf, J.C. Perruchetti, D. Bolmont et G. Gewinner, Phys. Rev. 44 (1991) 8407 et Phys. Rev. 44 (1991) 11472

[22] P. Krüger, O. Elmouhssine, J. C. Parlebas et C. Demangeat, Phys. Rev. 54 (1996) 6393

[23] Ch. Roth, Th. Kleeman, F. U. Hillebrecht et E. Kisker. Phys. Rev. B 52 (1995) R15691

[24] Ruqian Wu, A. J. Freeman, Phys. Rev. B 51 (1995) 17131

[25] A. Vega, S. Bouarab, H. Dreyssé et C. Demangeat, Thin Sol. Films 275 (1996) 103

[26] S. Handschuh et S. Blügel, submitted to Phys. Rev. Lett. (1996)

[27] S. Andrieu, P. Arcade, H.M. Fischer, M. Piecuch, M. Finazzi, F. Yubero, F. Chevrier, G. Krill et K. Hricovini, J. Magn. Magn. Mat. (1997) sous presse

[28] S. Andrieu, M. Finazzi, F. Yubero, H.M. Fischer, P. Arcade, F. Chevrier, L. Hennet, K. Hricovini, G. Krill et M. Piecuch, soumis Phys. Rev. Lett. (1996)

[29] O. Elmouhssine, G. Moraitis J. C. Parlebas et C. Demangeat, Phys. Rev. B 55 (1997) sous presse

[30] P. Grünberg, R. Schreiber, Y. Pang, M.B. Brodsky, H. Sowers, Phys. Rev. Lett. 57, 2442 (1986).

[31] Workshop "Fe/Cr interface Magnetism", Strasbourg, (juin 1996)

[32] S. Miethaner et G. Bayreuther, J. Magn. Magn. Mat. 148 (1995) 42

[33] D. Stoeffler et F. Gautier, J. Magn. Magn. Mat. 147 (1995) 260

[34] R. Lorenz et J. Haffner, J. of Phys., Condens. Matt.7 (1995) L253

[35] M. Freyss, D. Stoeffler et H. Dreyssé, Phys. Rev. B 54 (1996) R12677

[36] H. Dreyssé et C. Demangeat in Synchrotron Radiation and Magnetism ( E. Beaurepaire, B. Carrière et J.P. Kappler eds; Edition de Physique (Paris) 1997, sous presse) 